\title{
Frequency Controlled Noise Cancellation for Audio and Hearing Purposes
}

\author{
Ali O. Abid Noor \\ Depart of Communication Engineering, University of Technology-Iraq, \\ Baghdad, Iraq \\ Email: 30212@uotechnology.edu.iq
}

Received January 4, 2020; Revised April 17, 2020; Accepted May 17, 2020

\begin{abstract}
Methods for hearing aids sought to compensate for loss in hearing by amplifying signals of interest in the audio band. In real-world, audio signals are prone to outdoor noise which can be destructive for hearing aid. Eliminating interfering noise at high speed and low power consumption became a target for recent researches. Modern hearing compensation technologies use digital signal processing which requires minimum implementation costs to reduce power consumption, as well as avoiding delay in real time processing. In this paper, frequency controlled noise cancellation (FCNC) strategy for hearing aid and audio communication is developed with low complexity and least time delay. The contribution of the current work is made by offering a method that is capable of removing inherent distortion due filter-bank insertion and assigning adaptive filtering to a particular sub-band to remove external noise. The performance of the proposed FCNC was examined under frequencylimited noise, which corrupts particular parts of the audio spectrum. Results showed that the FCNC renders noise-immune audio signals with minimal number of computations and least delay. Mean square error (MSE) plots of the proposed FCNC method reached below -30 $\mathrm{dB}$ compared to $-25 \mathrm{~dB}$ using conventional sub-band method and to $10 \mathrm{~dB}$ using standard full-band noise canceller. The proposed FCNC approach gave the lowest number of computations compared to other methods with a total of 346 computations per sample compared to 860 and 512 by conventional sub-band and full-band methods respectively. The time delay using FCNC is the least compared to the other methods.
\end{abstract}

Keywords: Hearing aids, noise cancellation, adaptive filtering, filterbanks

\section{INTRODUCTION}

Amplifying audio signals in hearing aids is not the only target in improving hearing capabilities for people with hearing problems. Noise 
interfering from outside world is a serious threat to hearing and audio communication. This requires the use of special digital signal processing arrangements to overcome the problem. Conventionally, in hearing aids, the target audio signal is amplified to increase the strength of the signal as appropriate for the user. As a results, noise in a certain frequency band within the audio spectral-range also amplified, which adversely affects the quality of the target signal. In this case, the audio spectrum is divided into sub-bands in order to adjust the strength in certain parts of the audio spectrum to facilitate hearing aids [1]. However, amplifying the target signal is not always a successful option; therefore, sub-band adaptive filtering is a possible cure for the noise issue [2]. In this context, filter-banks are used to split the spectrum of the audio signal, and then using adaptive filters such as the normalized least mean square (NLMS) in sub-bands [3]. Nevertheless, signal splitting and reconstruction is encountered with inherent filter-bank distortion, this includes reconstruction distortion, which results from mismatching between the analysis and synthesis filters. Furthermore, subband adaptive filtering requires highly selective, causal, linear phase analysis filters to avoid aliasing distortion [4]. This can be achieved by using extremely high order finite impulse response FIR filters, which leads to realtime implementation problems, as well as intolerable signal delay. Therefore, the aim of the current research is to eliminate sub-band noise from audio signals with the lowest number of computations and acceptable time delay.

\section{RELATED WORKS}

Problems incorporating with using filter-banks for band-splitting have been treated in literature; issues such as aliasing distortion have been treated in [4], convergence rate of the adaptive filter have been dealt with in [5] and [6]. Computational complexity and input-output delay have been treated in [7] also in [8], and recently, reducing sub-band noise is targeted in [9]. These solutions to problems of adaptive filtering in sub-bands are depending on using FIR filters due to their stability and phase distortion free properties. However, this leads to realization problems in digital signal processing, since commonly used, moderate-price digital signal processors offered by manufacturers have limited computational and storage capacities.

Regarding adaptive filtering, the least mean square (LMS) filter is often involved in applications such as noise cancellation in telecommunication systems [10]. However, the application of this technique to audio noise cancellation requires extremely large number of computations. In addition, the convergence of the LMS filter responds very slowly to colored noise i.e. noise that is restricted to a limited band in the frequency spectrum of the input signal [10], [11]. These problems are of high importance in hearing aid, because it requires fast response at minimum processing power. Therefore, a number of solutions have been proposed in the last decade to overcome these issues. These include the use of infinite impulse response (IIR) adaptive filtering [12], [13], recursive least squares (RLS) adaptive filtering [14], [15] 
and block adaptive filtering [16], [17]. Although these solutions gave improvement in a particular side, they however lead to a worsening in some other sides. For example, the use of RLS filtering leads to large computational burden, block adaptive filters increases signal delay, while in the IIR adaptive filter solution the problem is related to phase distortion and instability, which is not acceptable in audio and hearing applications. In the following, the most related techniques of digital filtering for noise cancellation are described and discussed.

The general form of a digital filter is represented by the following transfer function in the $\mathrm{z}$-domain:

$$
H(z)=\frac{\sum_{n=0}^{N} b(n) z^{-n}}{1+\sum_{n=1}^{M} a(n) z^{-n}}=\frac{b_{0}+b_{1} z^{-1}+b_{2} z^{-2}+\ldots . b_{N} z^{-N}}{1+a_{1} z^{-1}+a_{2} z^{-2}+\ldots . a_{M} z^{-M}}
$$

where $n$ is a discrete time index, $N$ is the order of the numerator and $M$ is the order of the denominator; $(a)$ and $(b)$ are numerical coefficients. For finite impulse response filter (FIR), the denominator is set to 1 and the transfer function of the filter becomes:

$$
H(z)=\sum_{n=0}^{N} b(n) z^{-n}=b(0)+b(1) z^{-1}+b(2) z^{-2}+\ldots . b(N) z^{-N}
$$

This is a causal implementation of the filter and $b$ is replaced by $h$ :

$$
H(z)=\sum_{n=0}^{N} h(n) z^{-n}=h(0)+h(1) z^{-1}+h(2) z^{-2}+\ldots . h(N) z^{-N}
$$

In time domain, this is represented by the by the following:

$$
h(n)=[h(0) h(1) h(2) \quad \ldots . . h(N)]
$$

with $h(0), h(1), h(2)$ to $h(N)$ are numerical values called taps, coefficients, or weights of the filter, they depend on the discrete time $n$, which is related to the actual time $t$ via the sampling frequency $f_{s}$ by the following relationship:

$$
t=\frac{n}{f_{s}}
$$

by replacing $z$ with $e^{-j 2 \pi f}$ in Equation (3), the frequency domain representation of $h(n)$ is obtained as follows:

$$
H(f)=\sum_{n=-\infty}^{\infty} h(n) e^{-j 2 \pi n f}
$$


For IIR filter representation, we rewrite Equation (1), the general equation expressing the filter is:

$$
H(z)=\frac{b_{0}+b_{1} z^{-1}+b_{2} z^{-2}+\ldots . b_{N} z^{-N}}{1+a_{1} z^{-1}+a_{2} z^{-2}+\ldots . a_{M} z^{-M}}
$$

This represents a general IIR filter. Unlike FIR filter which only has a numerator, IIR filters are fully rational functions, they have numerators and denominators, meaning they have both poles and zeros. Therefore IIR filters are prone to instability because of the possibility the denominator collapses to zero. Also, IIR filters have non-linear phase response which causes distortion in the output signal. However, IIR filters have sharp frequency responses compared to FIR filters. The total coefficient count for IIR filters is $N+M+1$ which is needed to compute each new output. Normally, for few coefficients, IIR filter shows sharper response compared to FIR filter with higher number of coefficients. Figure 1 shows a comparison of IIR and FIR responses. In the case of FIR, large number of filter taps i.e. high order is required to achieve the same response as IIR filter. Therefore processing delay increases with filter order.
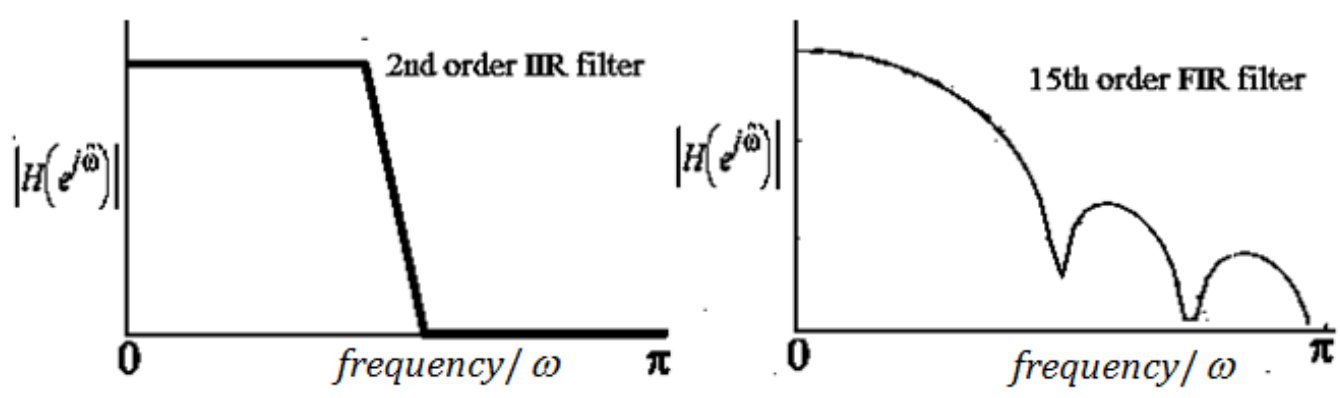

Figure 1. Response comparison of IIR and FIR filters

High order IIR filters are normally derived from second order all-pass sections [18]. A detailed discussion of FIR and IIR filters can be found in [19] and [20].

Adaptive filters are also digital filters, usually FIR type because of its guaranteed stability. However, an adaptive filter differs from a normal digital filter in that the coefficients of the filter are made to change values according to some algorithm. Figure 2 shows an adaptive filter based on FIR filter and the least mean squares LMS algorithm as the controlling algorithm. 


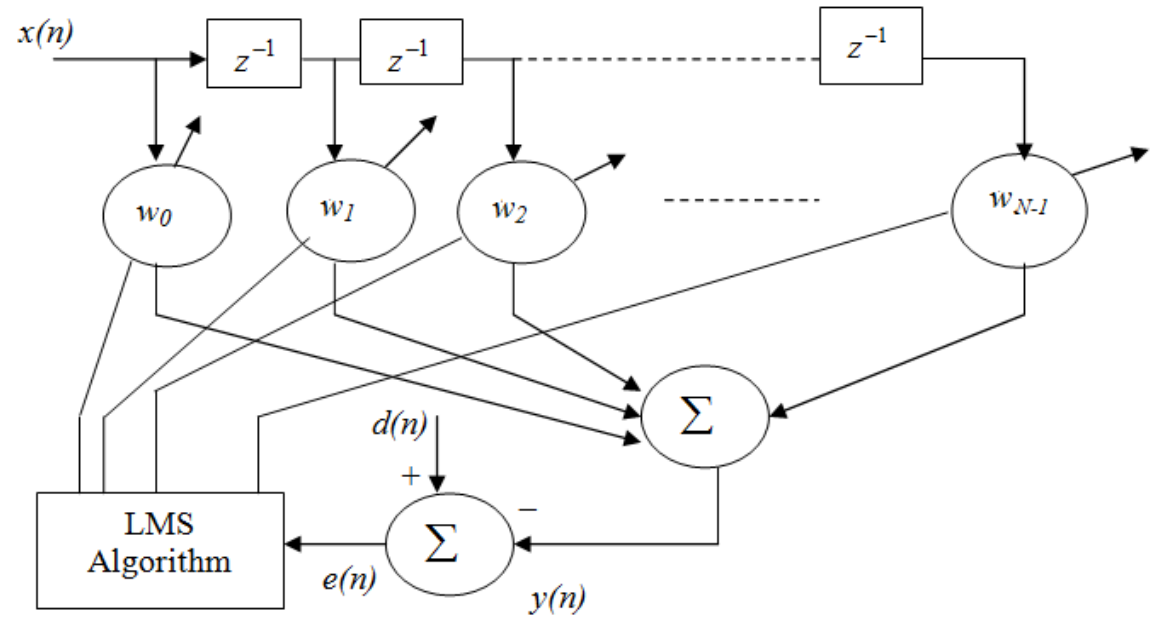

Figure 2. An adaptive filter using the LMS algorithm

The LMS is the most commonly used adaptive algorithm for its robustness and simplicity [10]. The operation of the LMS is described as follows. With reference to Figure 2, for each time index $(n)$ the weight coefficients $\mathbf{w}$ of the filter are changed according to the following set of equations:

$$
\begin{aligned}
& \mathbf{w}_{n+1}=\mathbf{w}_{n}+\mu \cdot \mathbf{x}_{n} \cdot e(n) \\
& \mathrm{e}(n)=d(n)-y(n) \\
& y(n)=\mathbf{x}_{n}^{T} \mathbf{w}_{n}
\end{aligned}
$$

with $\mathbf{x}_{n}$ is a vector of length $L$ representing the input data, $\mathbf{w}_{n}$ is the adaptive filter weight vector at discrete time $n, y(n)$ represents the output of the filter at any instant of time $n, d$ is the desired input, $\mu$ is a fixed step size and $T$ is a transpose operator.

The normalized LMS (NLMS) algorithm is a modification of the LMS algorithm, in which the step size that is inversely proportional to the input signals energy. Hence the update equation compared with (7) becomes:

$$
\mathbf{w}_{n+1}=\mathbf{w}_{n}-\hat{\mu} \cdot \mathbf{x}_{n} \cdot e(n)
$$

where:

$$
\hat{\mu}=\left(\mu /\left(\left\|\mathrm{x}_{\mathrm{n}}\right\|^{2}\right)\right.
$$

The value of $\mu$ is between 0 and 2 and $\left\|\mathrm{Xn}_{\mathrm{n}}\right\|$ is the norm or power of the input vector $\mathbf{x}_{n}$. For audio applications considered in this paper the normalized version of the LMS algorithm is used. Compared to the basic LMS algorithm, the step-size $\hat{\mu}$ is made variable, thus providing better tracking capability. The number of computations required by the NLMS is proportional to $N$ the filter's length or order. In audio applications $N$ has to be very large which results in large number of computations per unit sample, as well as large group delay, which is undesirable and causes problems in real- 
time implementation. For this reason sub-band adaptive filtering is used to overcome these problems.

In sub-band adaptive filtering the input signal is divided into smaller bands and shorter adaptive filters are used in each sub-band as shown in Figure 3. The analysis filter bank decomposes the input signal into many sub-bands using a parallel set of band-pass filters. Similarly, the synthesis bank reconstructs the sub-band signals using a set of parallel filters. Between analysis and synthesis stages adaptive filtering is performed. To preserve the same number of samples, down samplers and up samplers are used in the configuration [21]. Compared to the full band version, the sub-band adaptive filters shall be shorter in length hence lower complexity and shorter delay. However, these advantages of the sub-band configuration are offset by the inherent distortion introduced by analysis filters insertion. For good performance, high order analysis filters are required for signal separation, this leads to large number of computations.

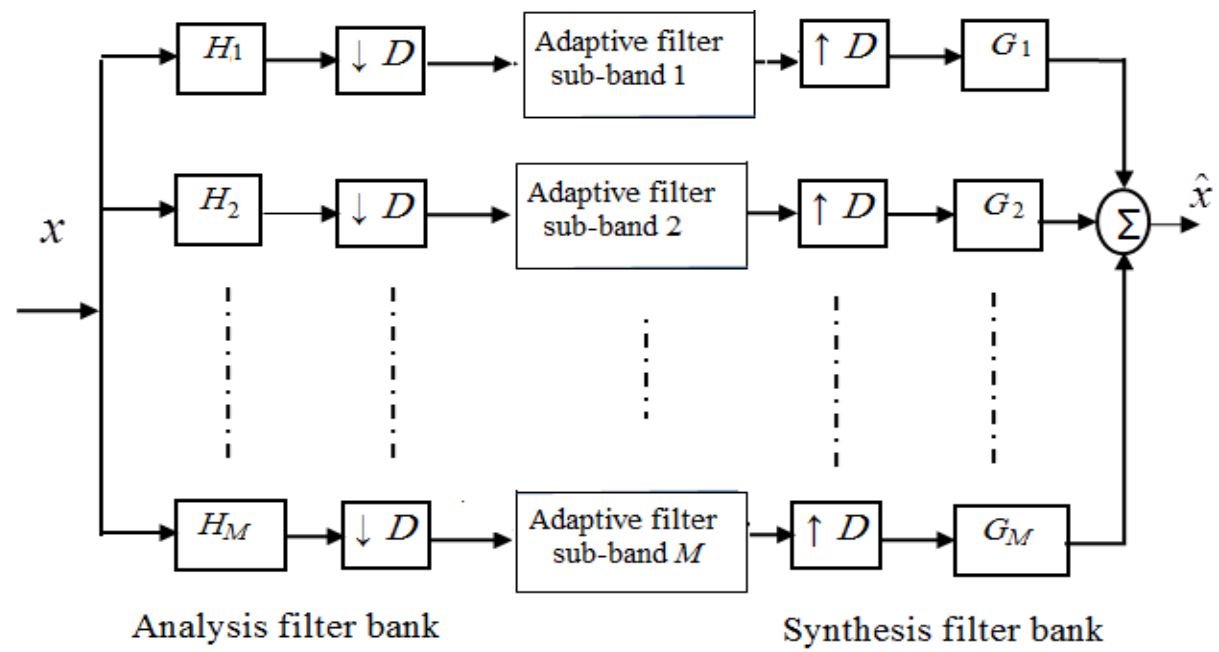

Figure 3. Sub-band adaptive filtering

In sub-band signal splitting, it is not necessary to design each filter in the analysis filters, an efficient implementation is to use DFT modulated filter bank. The $M$ sub-band filters of a DFT modulated filter bank are derived by frequency shifting a well-designed low pass prototype filter $h_{0}$ of length $N$ in the following way:

$$
\begin{aligned}
& h_{k}(n)=h_{0}(n) e^{-2 \pi k / M} \\
& \text { for, } \mathrm{O} \leq k \leq M-1, \mathrm{O} \leq n \leq N-1
\end{aligned}
$$

The number of computations requires for the sub-band scheme is constituted of the cost of the prototype analysis filter plus the cost of the DFT transform plus the cost of the sub-band adaptive filter plus the cost of the synthesis filters. Also the group delay is determined in the same way. The sub-band implementation using FIR filters demands long filter lengths in the analysis stage to achieve acceptable signal separation and hence efficient 
adaptive filtering in sub-bands. Furthermore, the adaptive filters in all subbands are operated at the same time even if the noise is limited to one of the sub-bands only. Therefore a frequency controlled noise canceller FCNC is developed in this paper to overcome problems associated with both full band and sub-band schemes.

\section{ORIGINALITY}

This research focuses on cancelling band-restricted external noise in audio signals with two-fold objective, first is to use low complexity analysissynthesis filters having reduced reconstruction distortion. This is achieved through the use of infinite impulse response filters at the analysis stage, with a special relationship between the analysis and synthesis filters, in which the distortion is minimized implicitly within the synthesis filters. The second is to use frequency-controlled adaptive filter that is activated in the noisy subband rather than the whole audio range. Thus, using this combined approach; better noise reduction performance is achieved at a lower number of computations than conventional methods. In the proposed solution, the subband filter is much shorter than the full-band one, while the analysissynthesis filters possess minimum effect on the reconstructed signal.

\section{SYSTEM DESIGN}

The first step in the proposed approach is to split the incoming audio signal into sub-bands. This is achieved by using analysis filter-bank. The analysis filter-bank is derived from a second order all-pass filter. The constraint here is to keep the computational cost and signal delay as minimum as possible. The second order all-pass prototype analysis filter is described by the following equations:

$$
\begin{aligned}
& H(z)=\frac{1}{2} \sum_{k=0}^{N-1} A_{k}\left(z^{-2}\right) z^{k} \\
& A_{k}\left(z^{2}\right)=\prod_{n=1}^{L} A_{k, n}\left(z^{2}\right)=\frac{\alpha_{k, n}+z^{-2}}{1+\alpha_{k, n} z^{-2}}, \quad k=0,1
\end{aligned}
$$

where $\alpha_{k, n}$ is the coefficients of the $k^{\text {th }}$ all-pass section in the $n^{\text {th }}$ branch, $L$ is the number of sections in the $n^{\text {th }}$ branch and $N$ is the total number of branches in the structure. The parameters in the above equations are determined by filter specifications. The second order all-pass filter is used as a prototype from which all filters in the analysis filter-bank are derived. For fixed point DSP implementation, it is useful to use cascaded first or second order filters to produce other higher order filters. In this work, these filters are used to produce filter-banks with improved filtering quality. The highpass and low-pass filters form two-band analysis filter-bank are described in poly-phase form by the following equations. 


$$
\begin{aligned}
& H_{0}(z)=\frac{1}{2}\left(A_{0}\left(z^{2}\right)+z^{-1} A_{1}\left(z^{2}\right)\right) \\
& H_{1}(z)=\frac{1}{2}\left(A_{0}\left(z^{2}\right)-z^{-1} A_{1}\left(z^{2}\right)\right)
\end{aligned}
$$

These filters are band-limiting filters representing low-pass $H_{0}(z)$ and high-pass $H_{1}(z)$ as shown in Figure 4. The poly-phase implementation reduces the implementation costs to a half of the original implementation, where $A_{0}(z)$ and $A_{1}(z)$ are causal, real, stable all-pass filters. This implementation is modified by shifting the down-sampler to the input to give more efficient implementation using the noble identities for multi-rate systems [21]. This result in a small number of calculations required per filter order, and very high filtering performance. Such a structure is required for filtering with high speed of operation. A binary tree form based on equations (16) and (17) is used for the analysis filter bank implementation.

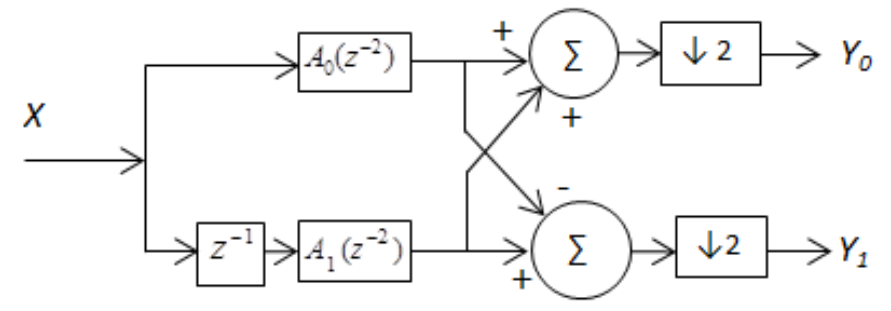

Figure 4. Poly-phase structure of the analysis filter-bank

The analyzed audio signal is then filtered in sub-band treating each subband in the analysis stage as an independent band of frequency. The subband signals are required to be recombined to form the final reconstructed signal after removing external noise. The main issue in the reconstruction process is resulted from the insertion of the analysis filter-bank which produces aliasing distortion. The input-output reconstruction is required to be reduced by a perfect reconstruction filter-bank. Since there is no such a filter-bank that can be realized, an optimization procedure is adopted in this research to reduce filter-bank errors. Starting by representing the analysis prototype filter $H(z)$ in the frequency domain, given that $\phi$ is the phase response of the analysis filter, then it can be expressed as:

$$
H\left(e^{j \omega}\right)=\left|H\left(e^{j \omega}\right)\right| e^{j \phi(\omega)}
$$

To reduce reconstruction error, the prototype synthesis filter is implemented using FIR filter, the desired frequency response of which is related to analysis one by the following:

$$
G_{d}\left(e^{j \omega}\right)=\left|G\left(e^{j \omega}\right)\right| e^{j \beta}=\left|H\left(e^{j \omega}\right)\right| e^{-j|\omega . \partial+\phi(\omega)|}
$$

where $\beta$ is the phase response of the synthesis filter, $\partial$ is the group delay. This arrangement is capable of equalizing the distortion introduced by the analysis filter-bank. The evaluation of the coefficients of the prototype 
synthesis filter is achieved by minimizing the square of the error using a weighting function $\hat{w}(\omega)$ as follows:

$$
\underset{\min }{L S}=\sum \hat{w}(\omega)\left|G\left(e^{j \omega}\right)-G_{d .}\left(e^{j \omega}\right)\right|^{2}
$$

An approximation of the desired frequency response of the synthesis filters is obtained by truncating the impulse response of the desired prototype filter and transferring to the frequency domain. This minimizes the errors due to aliasing, amplitude and phase in the filter-bank. Hence an approximate perfect reconstruction is achieved with the output is merely a delayed version of the input. The number of computations due to the implementation of the synthesis filters $G_{k}(z)$ is reduced to a half by utilizing poly-phase form of the filters, using type-2 poly-phase implementation. Poly-phase implementation of the synthesis filters is given by the following:

$$
G(z)=\sum_{k=0}^{M-1} z^{-(M-1-k)} G_{k}\left(z^{M}\right)
$$

The optimized analysis-synthesis filter-bank offers a better input-output relationship in which the effect is a delay with minimum distortion. This way, artifacts due filter-bank insertion are eliminated.

The second part of the proposed design procedure focuses on removing external environmental noise using adaptive filtering with minimum implementation costs. The procedure followed here is to use frequency controlled adaptive filtering. Once the corrupted audio input signal is split into sub-bands, a threshold is used to locate the noisy sub-band, then an adaptive filter is activated in that particular sub-band to remove noise from it. Therefore, instead of activating adaptive filters in the whole audio range, a shorter adaptive filter is activated in the corrupted part of the audio band, resulting in faster response due to whiter spectrum, as well as lower computational burden than the full-band implementation. In the following a description of the procedure: The output from the noisy part in the analysis filters is subjected to an adaptive filter in the $k^{\text {th }}$ sub-band, with the normalized least square NLMS algorithm as the controlling algorithm of the adaptive process. The NLMS adaptation in any of the sub-bands is described by the following set of equations in the discrete time domain $n$ :

$$
\begin{aligned}
& \mathbf{w}_{k}(n+1)=\mathbf{w}_{k}(n)+\mu e_{k}(n) \mathbf{x}_{k} \\
& e_{k}(n)=d_{k}(n)-y_{k}(n)
\end{aligned}
$$

for any $k^{\text {th }}$ sub-band within M sub-bands

where $\mathbf{w}$ is the weight coefficients vector of the adaptive filter, $\mu$ is the normalized adaptation step size, $\mu$ has to be divided by the energy of the input noise vector $x_{k}, e_{k}$ is the branch error, $d_{k}$ is the desired input, $y_{k}$ is the branch output of the adaptive filter. Let the input audio signal be $s$, in $z-$ domain, Equation (23) is expressed in the following: 


$$
\begin{aligned}
& E_{k}(z)=D_{k}(z)-Y_{k}(z) \\
& D_{k}(z)=H_{k}(z) S(z) \\
& Y_{k}(z)=H_{k}(z) X(z) W_{k}(z)
\end{aligned}
$$

The error signals from the noisy sub-band as well as signals from clean subbans are interpolated by the synthesis FIR filters $G_{k}(z)$. Assuming the branch adaptive filter from the noisy band has reached full convergence and the output signal is a delayed version of the input signal. The input-output relationship can be expressed by the following;

$$
\sum_{k=0}^{M-1} G_{k}(z) E_{k}(z)=c z^{-\partial} S(z)
$$

where $c$ is a constant, $\partial$ is the total delay of the output and $k$ is the sub-band index. This is a perfect reconstruction relationship. By accepting very small amplitude errors, the processed signal can be expressed by the following:

$$
\hat{S}(z) \approx c z^{-\partial} S_{k}(z)
$$

In discrete time domain, Equation (28) above is expressed as:

$$
\hat{s}(n) \approx \operatorname{cs}(n-\partial)
$$

Optimizing the filter-bank in the described procedure and activating adaptive filter in the noisy sub-band reduces internal and external noise greatly at the same time. It also reduces the implementation cost by using fewer computations, thus saving battery life in hearing aids and hand-free communication. The optimization process does not consume computations in the noise cancellation process because it is performed off-line. The implementation costs of the analysis filter-bank are negligible since it is based on all-pass IIR filters with poly-phase implementation. The main contribution of the implementation costs comes from the adaptive filter and the synthesis FIR filters. However, these costs are kept below certain level in such a way it possess no effect on hearing delay. Delays of more than $30 \mathrm{~ms}$ can cause mismatch between hearing and visual information [22]. The noise cancellation process is described as follows.

After splitting the input signal into several frequency sub-bands, the average level of the desired signal in each sub-band is compared to a stored threshold. If the level of the signal exceeds this threshold then the adaptive filter in that particular frequency band is activated to remove the noise, thereafter combining with other sub-bands to produce the final signal. The threshold is obtained by calculating the average level of the user voicetemplate, which is assumed to be available beforehand. The flow chart in Figure 5 below describes the procedure: 


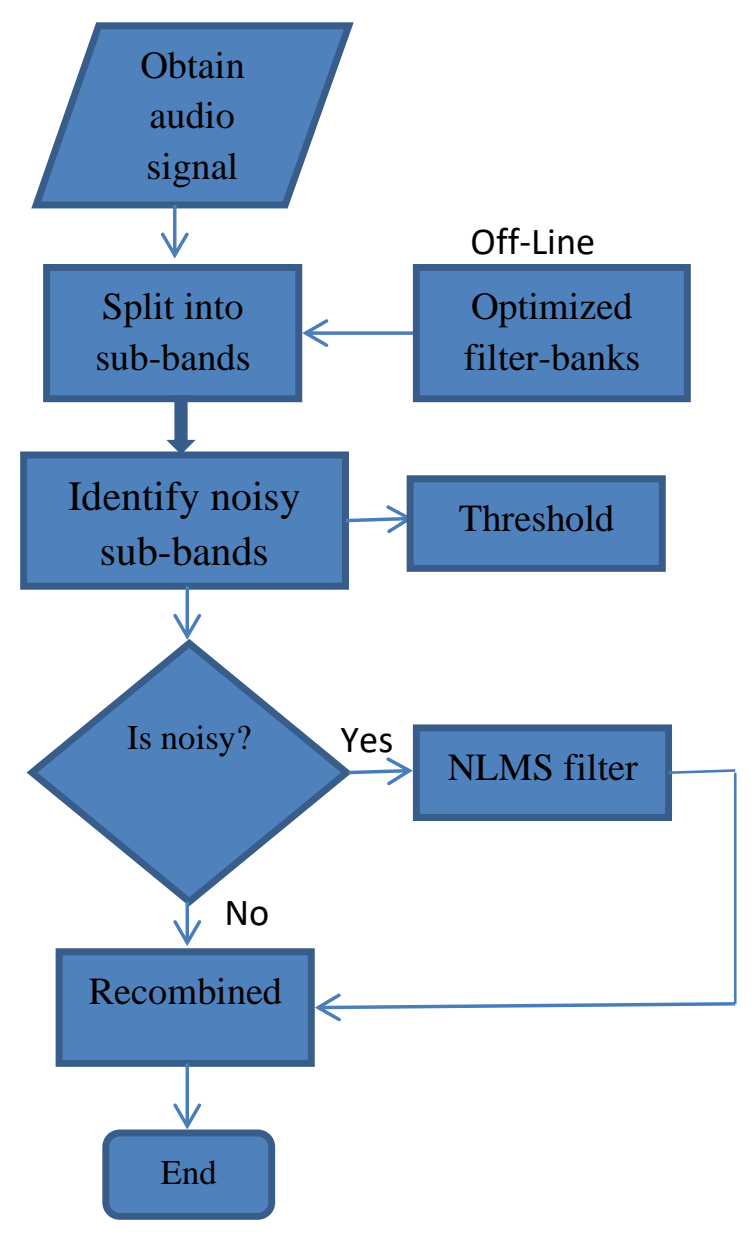

Figure 5. Flow chart of the proposed procedure

\section{EXPERIMENT AND ANALYSIS}

To test the performance of the proposed frequency controlled noise canceller (FCNC), a clean chirp signal sweeping in the audio range between 0 and $8 \mathrm{kHz}$ is used as the target signal. This signal is generated to simulate an audio signal that can excite the whole range of interest in hearing aids. The test signal is sampled at $16 \mathrm{kHz}$; a portion of this signal is shown in Figure 6 alongside with random noise that is used to simulate environmental noise. The type of noise used in the experiments is normally distributed pseudorandom whit noise with zero mean and variance of 0.99 . The level of noise is $0 \mathrm{~dB}$ signal to noise ratio. This means that the signal is totally buried with noise which is a sever noise condition.

It is thought that the choice of the chirp as the test signal is ideal for audio range testing, since using a speech signal can have components only below $4 \mathrm{kHz}$. However, experiments using speech signal will also be provided to prove the validity of the proposed approach in real world environment. The spectrum of the chirp signal is projected in Figure 7. 

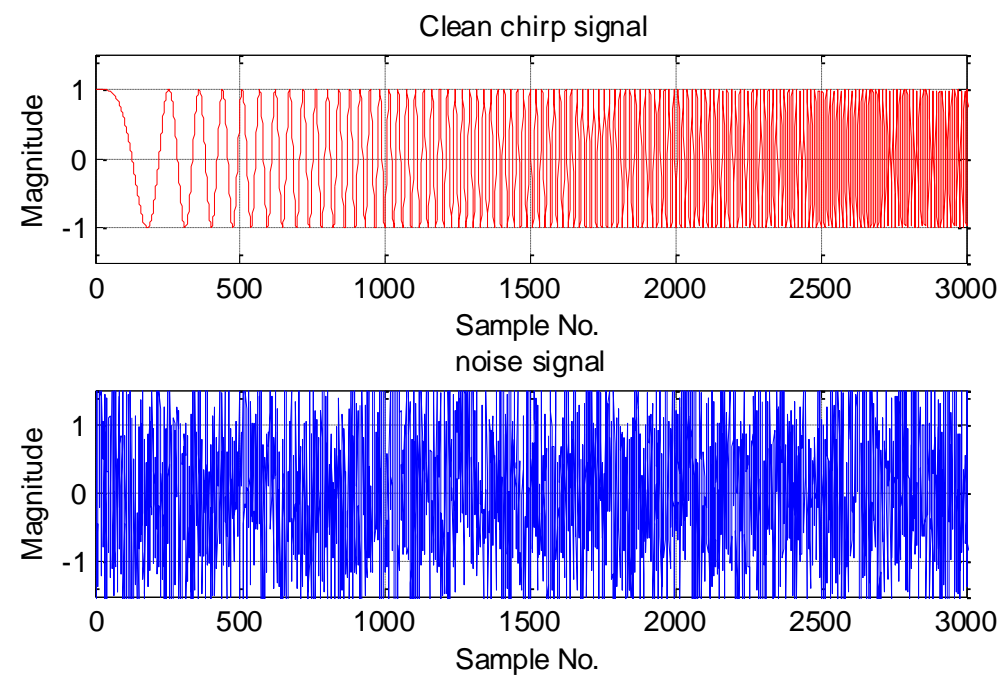

Figure 6. Part of the test signal (top) and white noise (bottom)

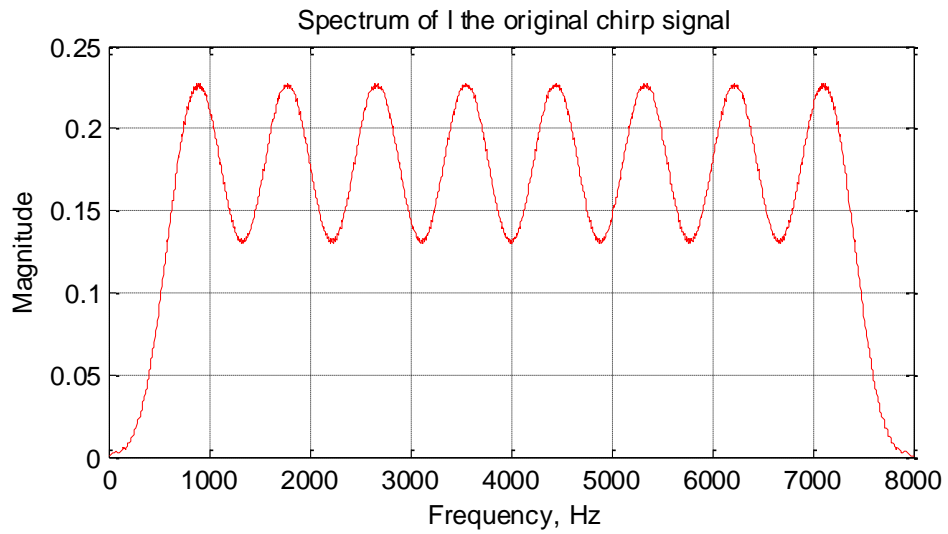

Figure 7. Frequency spectrum of the chirp signal

The frequency spectrum of the test chirp signal is divided into 8 equallyspaced sub-bands, using analysis filter-bank based on a prototype 8th order IIR filter, implemented in a poly-phase form. The magnitude response of the analysis filter bank is shown in Figure 8. 


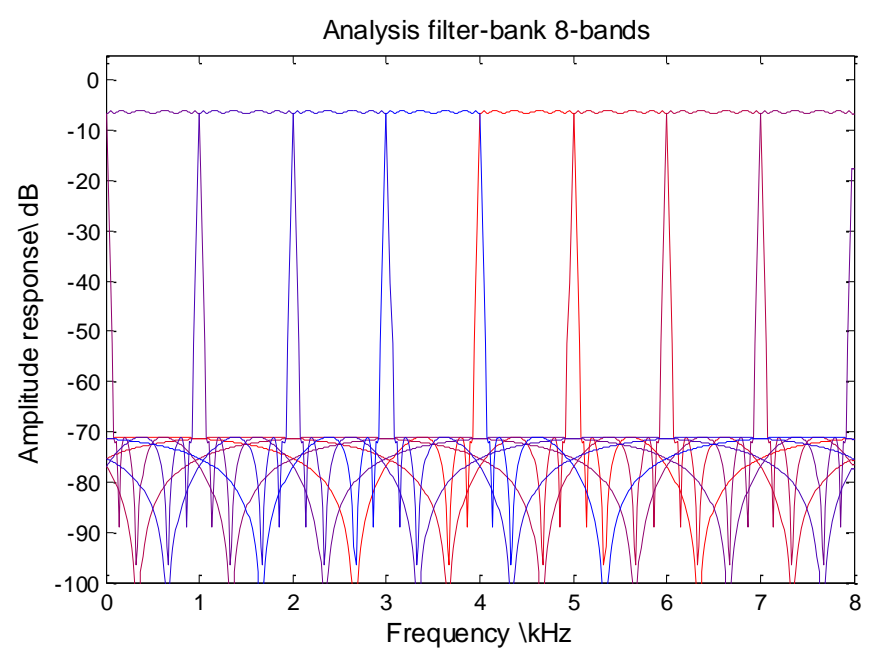

Figure 8. Amplitude response of the analysis filters

The synthesis filters are related to the analysis filters according to Equation (19). The optimizing of the prototype synthesis filter is accomplished according to Equation (20), Figure 9 shows minimizing the error of the weighted least squares which renders the lowest input-output distortion.

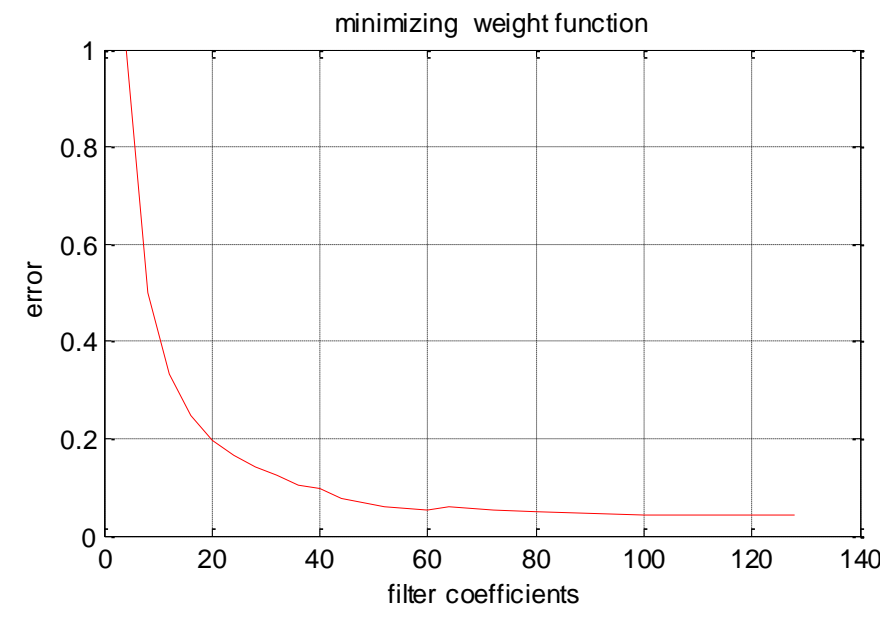

Figure 9. Minimizing the error of the weighted least squares of the synthesis prototype filter

The frequency response of the synthesis filter-bank which is based on DFT modulated FIR prototype filter is shown in Figure 10. 


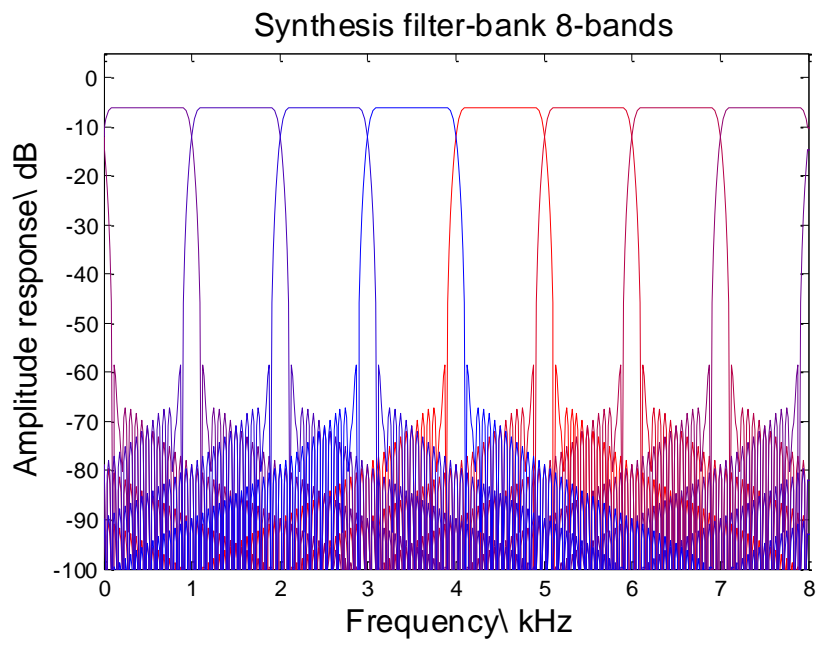

Figure 10. Amplitude response of the synthesis filters

The distortion is minimized as shown in Figure 11 and the distortion due filter-banks is shown in Figure 12.

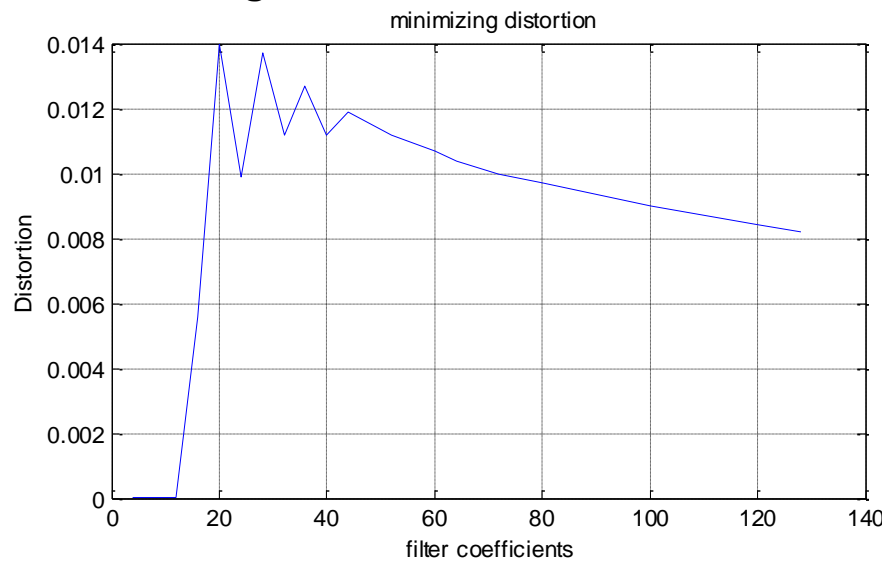

Figure 11. Minimization of distortion due filter-banks

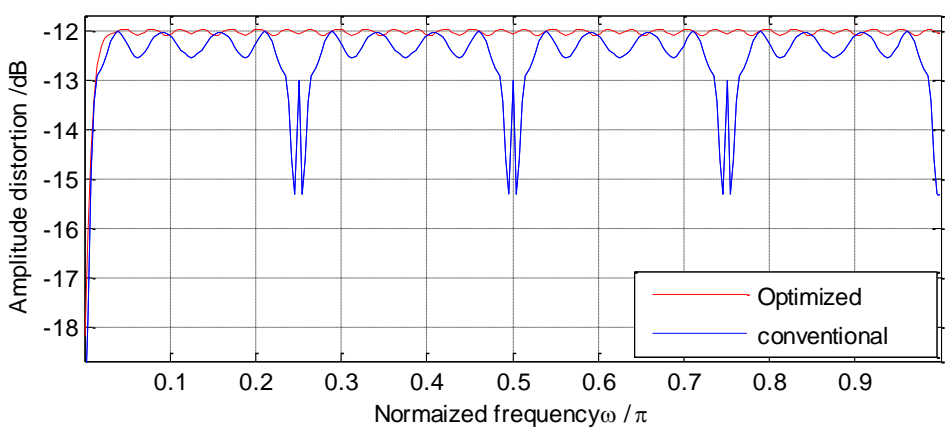

Figure 12. Amplitude distortion of the optimized filter-bank

Having performed the optimization of the analysis-synthesis filters, the structure is now ready for adaptive filtering. Adaptive filtering is based on using the NLMS algorithm which controls a 64-tap FIR filter. The fixed step size $\mu$ is set to 0.02 , this choice was based on previous experience with 
acoustic noise control. The quality of noise cancellation is measured by using the mean square error (MSE) of the adaptive filter's output. Noise cancellation performance of the proposed approach is examined under subband noise. The sub-band noise is obtained by passing the white noise in Figure 6 through band-pass filters with the same specifications as mentioned before. The output of the band-pass filters is shown in Figure 13. The subband noise is made to contaminate specific parts of the test signal as shown in Figure14.
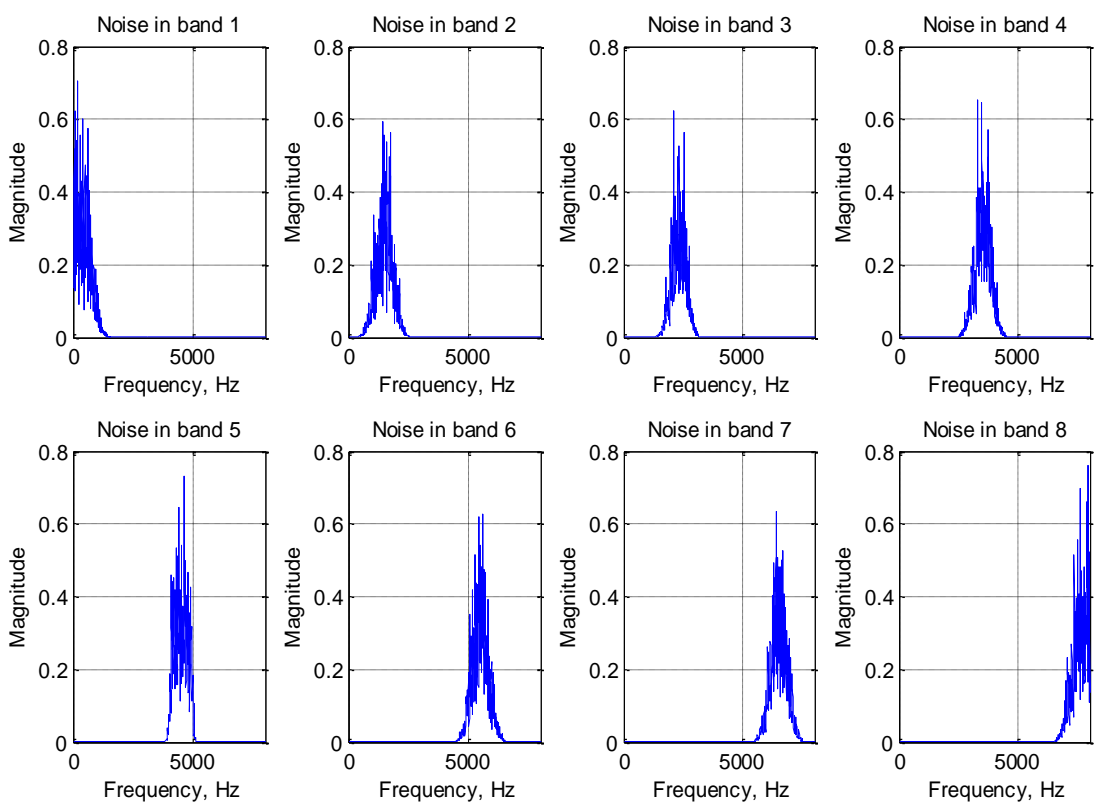

Figure 13. Generated noise in sub-bands of the audio range
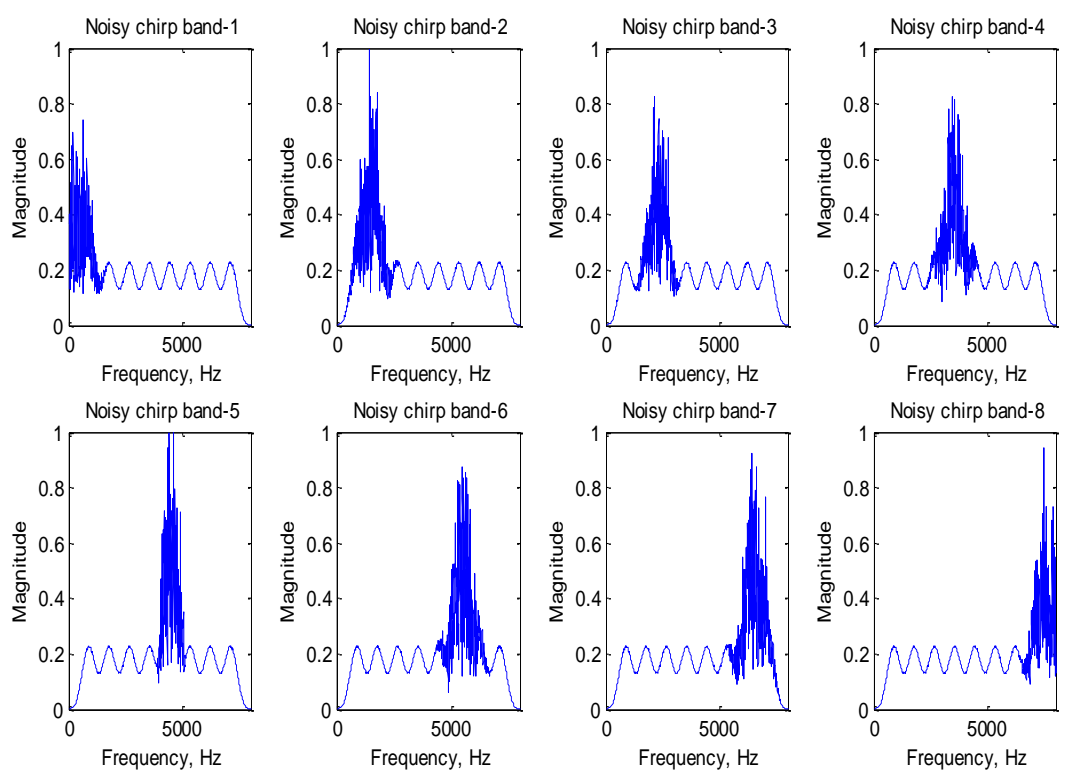

Figure 14. Noise corrupting sub-bands of the spectrum of the audio signal 
When the noise is interfering with a certain sub-band, the NLMS is ordered to run in that band only. This is done according to a preset noise threshold, hence cleaning that particular frequency-band from noise. Sometimes more than one sub-band is subjected to noise; in this case the adaptive filter is made to run in those corrupted sub-bands at the same time. However, acoustic noise is usually restricted to a certain band in the audio frequency range.

To analyze the overall performance of the proposed FCNC scheme, mean square error (MSE) plots are produced for cases of noise interfering with one band or two. The target audio signal of Figure 6 is subjected to noise restricted to sub-band 1 and used as the desired input to the noise canceller, while noise in that sub-band is applied to the reference input of the adaptive filter. Samples of MSE are obtained and smoothed using a moving average filter as shown in Figure 15. A comparison is made with a full-band NLMS as well as with a conventional sub-band scheme on the same graph.

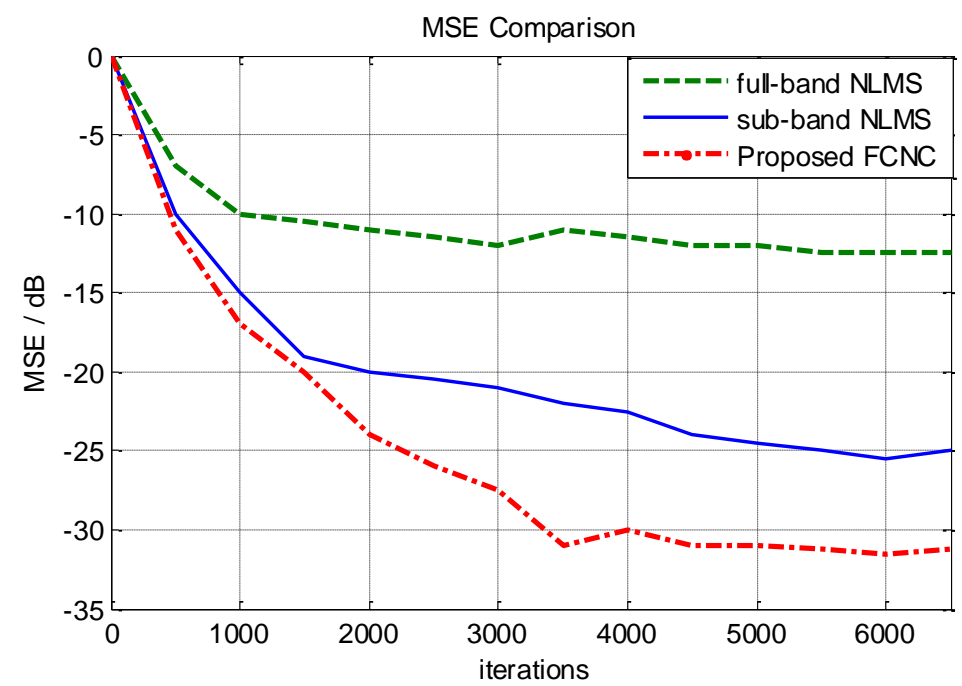

Figure 15. MSE comparison of the proposed FCNC with existing schemes

To be more realistic, the proposed FCNC is subjected to real world speech in the form of the utterance "Cottage cheese with chins is delicious" corrupted with noise in one or two sub-bands. In the first case, sub-band 1 is subjected to noise. Figure 16 shows the utterance before and after filtering by the FCNC. The performance is even better in sub-band-2 and higher as shown in Figure 17 for the case of sub-band 3, because the speech is more concentrated in the lower part of the frequency range. 


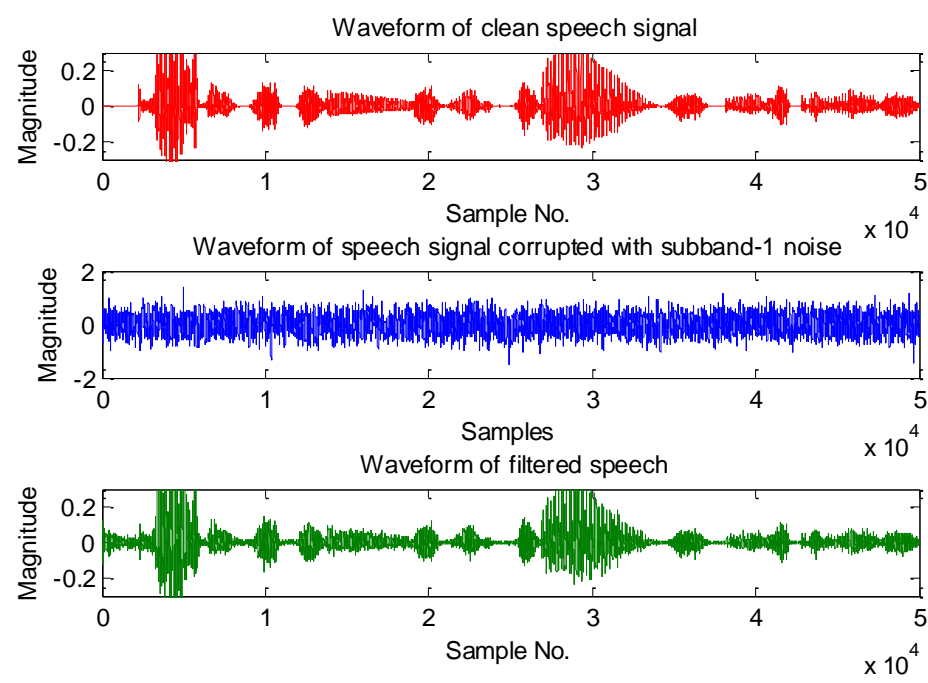

Figure 16. Performance of the FCNC with noise corrupting sub-band1
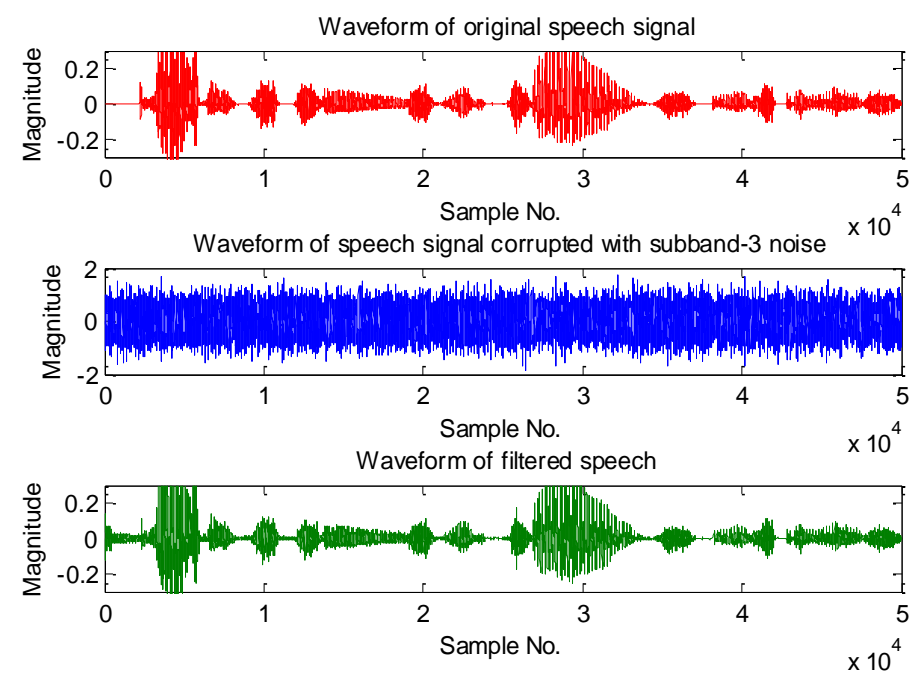

Figure 17. Performance of the FCNC with sub-band 3 subjected to noise

In the case where two sub-bands corrupted with external noise is also examined, two sub-bands are subjected to external noise, namely sub-band 1 and sub-band 3 and the run was repeated, Figure 18 depicts the process of the speech signal filtering. Several runs were conducted for other sub-bands and similar results were obtained. It is worth mentioning here that speech signals rarely have spectral components above $3.5 \mathrm{kHz}$, therefore it is not useful to repeat experiments on sub-bands higher than $4 \mathrm{kHz}$ i.e. sub-bands 5 and above. However, the design of $8 \mathrm{kHz}$ structure is advantageous in hearing aids, since audio range in real world may extend to more than $4 \mathrm{kHz}$ to contain music rhythm, sirens and similar high frequency audio signals [23]. 


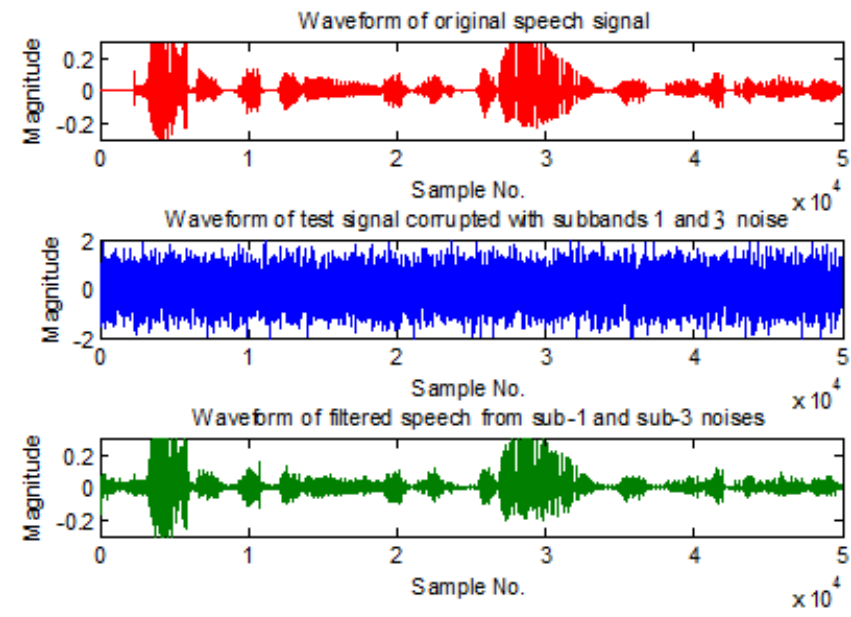

Figure 18. Sub-bands 1 and 3 are subjected to noise

To confirm the success of the proposed FCNC method, frequency spectrum graphs were also obtained to demonstrate the ability of removing noise components from the target speech signal as shown in Figure 19.
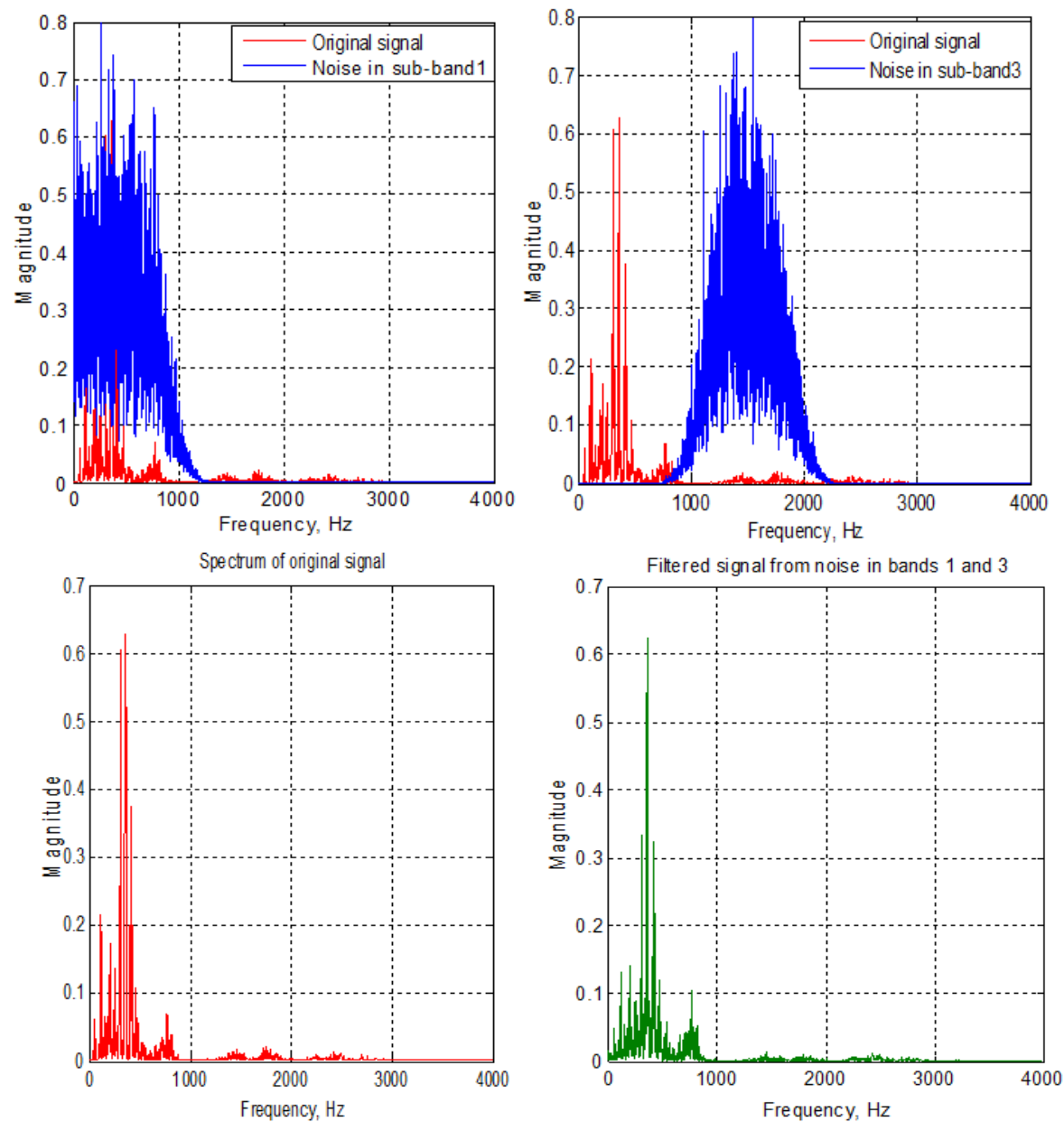

Figure 19. Frequency spectrums before and after filtering using FCNC 
It is clear from MSE plots in Figure 15 and from filtered signals shown in Figures 16, 17 and 18, also from the frequency spectrums for the signal before and after filtering in Figure 19, that the propose FCNC noise canceller with optimized filter-banks gives better noise cancellation performance than both full-band and the conventional sub-band noise cancellers. Referring to Figure 15, the full-band NLMS converges slowly with high level of steady state errors when the noise is restricted to a specific band. This is an expected result for the NLMS because the sub-band noise is colored for which the NLMS performs badly. Close inspection of the MSE plots of the proposed FCNC shown in Figure 15 reveals that the adaptation speed is better than the equivalent sub-band structure. This improvement resulted from using analysis filter-bank with steeper transition bands, good channel separation and very flat pass-band response within each sub-band. Thanks to the optimization of the analysis-synthesis filters, very small reconstruction errors i.e. near perfect was obtained. For sampling frequency of $16 \mathrm{kHz}$, the adaptation of the FCNC approaches $95 \%$ in less than 0.187 seconds.

The proposed FCNC approach also possesses the advantage of the lowest number of computations performed during one input sample. The equivalent DFT modulated FIR filter-bank that uses a 128-tap FIR filter, for 8band filter-bank renders an overall number of multiplications of 796 for subband analysis-synthesis filtering. In comparison, the use of the all-pass reduces the number of multiplications per input sample at the analysis stage to 24 and to a half of that in poly-phase decomposition. As far as adaptive process is concerned, the implementation costs around 64 per unit sample, the implementation cost of the synthesis filter-bank which is based on 128tap FFT modulated filter-bank is 128 plus the implementation of the FFT which costs about $\mathrm{N}^{*} \log (\mathrm{N})$ where $\mathrm{N}$ is the filter's order. Table- 1 shows the implementation cost and delay comparisons with full-band and conventional sub-band structures.

The main advantage of the proposed FCNC is the fast and better noise cancellation behavior. Regarding the audio band, the detection of whereabouts of noise is very important since it enables a short-length adaptive filter to work on nearly white noise in a particular sub-band of the audio spectrum, which in the case of NLMS renders fast convergence with very low steady state error at a lower number of computations.

Table 1. Computational costs and delay comparison

\begin{tabular}{|c|c|c|c|c|}
\hline Method & $\begin{array}{c}\text { Filter-bank } \\
\text { computation }\end{array}$ & $\begin{array}{c}\text { Adaptive } \\
\text { filter } \\
\text { computation }\end{array}$ & Overall costs & Delay/ms \\
\hline Full-band & 0 & 512 & 512 & 32 \\
\hline $\begin{array}{c}\text { Conventional } \\
\text { sub-band }\end{array}$ & 796 & 64 & 860 & 54 \\
\hline $\begin{array}{c}\text { Proposed } \\
\text { FCNC }\end{array}$ & 282 & 64 & 346 & 21 \\
\hline
\end{tabular}




\section{CONCLUSION}

According to results obtained from running the proposed FCNC with other existing structures, improved, low-complexity noise cancellation performance can be obtained from the procedure adopted in this research for audio communication and hearing purposes. The minimization of filter-bank errors in conjunction with a threshold controlled adaptive filter, gave a desirable noise cancellation performance as well as low signal processing delay that is necessary to be kept below some limits in hearing purposes. The convergence of the FCNC approaches more than 95\% in less than 0.187 seconds, which is very fast compared to full-band model. The proposed FCNC offers a decrease in the number of operations with minimum time delay.

\section{REFERENCES}

[1] N. S. Jensen, O. Hau, J. B. B. Nielsen, S. V. Legarth, Perceptual Effects of Adjusting Hearing-Aid Gain by Means of a Machine-Learning Approach Based on Individual User Preference, Trends in Hearing Vol. 23: pp.1-23. 2019.

[2] K. A. Lee, W. S. Gan, S. M. Kuo, Subband Adaptive Filtering: Theory and Implementation, John Wiley \& Sons Ltd (Wiltshire), pp.99-129. 2009.

[3] M. Djendi, A. Sayoud, A New Dual Subband Fast NLMS Adaptive Filtering Algorithm for Blind Speech Quality Enhancement and Acoustic Noise Reduction, Int. Journal of Speech Technology, Vol. 22, No.2, pp. 391-406. 2019.

[4] S. G. Kim, C.D. Yoo, T.Q. Nguyen, Alias-Free Subband Adaptive Filtering With Critical Sampling, IEEE Transactions on Signal Processing, Vol. 56, No. 5, pp.1894-1904. 2008.

[5] H. Choiand, H. D. Bae, Subband Affine Projection Algorithm for Acoustic Echo Cancellation System, EURASIP Journal on Advances in Signal Processing, Vol. 2007, Article ID 75621, doi:10.1155/2007/75621. 2007.

[6] K. A. Lee, W. S. Gan, Improving Convergence of the NLMS Algorithm Using Constrained Subband Updates, IEEE Signal Processing Letters, Vol. 11, No.9, pp. 736-239. 2004.

[7] M. R. Petraglia, P. Batalheiro, Non-Uniform Subband Adaptive Filtering With Critical Sampling, IEEE Transactions on Signal Processing, Vol. 56, No. 2, pp.565-575, 2008.

[8] C. Schüldt, F. Lindstrom, I. Claesson, A Low- Complexity Delayless Selective Subband Adaptive Filtering Algorithm, IEEE Transactions on Signal Processing, Vol. 12, pp.5840-5850, 2008.

[9] A. O. A. Noor, I. H. Al-Hussaini, S. A. Samad, Adaptive Cancellation of Localised Environmental Noise, Jurnal Kejuruteraan, Vol. 30, No. 2, pp.179-186, 2018.

[10] S. Haykin, Adaptive Filter Theory. Prentice Hall (New Jersey), Ed.5, pp. 160-187, 2013. 
[11] R. M. Ramli, A. O. A. Noor, S. A. Samad, Noise Cancellation Using Selectable Adaptive Algorithm for Speech in Variable Noise Environment, International Journal Speech Technology, Vol. 20, No. 3, pp. 535-542, 2017.

[12] P. S .R. Diniz, Adaptive IIR Filters. In: Adaptive Filtering. Springer (Boston MA), pp. 411-466, 2013

[13] M. Radenkovic, T. Bose, Adaptive IIR Filtering of Non Stationary Signals, Elsevier Signal Processing, Vol. 81(2001), pp. 183-195. 2001.

[14] Y. Yu, L. Lu, Z. W. Zheng, Y. Zakharov, R. C. de Lamare, Robust DCDBased Recursive Adaptive Algorithms. IEEE Transactions on Circuits and Systems II. DOI: 10.1109/TCSII.2019.2936407, 2019.

[15] B. K. Das, M. Chakraborty, Improved I O -RLS adaptive filter. Electronics Letters, Vol. 53. No 25, pp.1650. D0I: 10.1049/el.2017.3441, 2017.

[16] M. Narasimha, Block Adaptive Filter With Time-Domain Update Using Three Transforms, IEEE Signal Processing Letters, Vol. 14, No.1, Jan 2007, pp. 51-53, 2007.

[17] J. Liu, S.L. Grant. Proportionate Adaptive Filtering for Block-Sparse System Identification, IEEE/ACM Transactions on Audio, Speech and Language. Volume 24 Issue 4. Pp. 623-630, 2016.

[18] S. J. Schlecht, Frequency-Dependent Schroeder Allpass Filters, Journal of Applied Sciences (MDPI), 10, 187, pp1-11, 2019.

[19] K. D. Rao and M.N. Swamy, Digital Signal Processing. Springer Nature Singapore Pte Ltd. Chapter 11, pp693, 2018.

[20] G. Blanchet and M. Charbit, Digital Signal and Image Processing using MATLAB, ISTE USA, pp114-150, 2006.

[21] L. Milić, Multirate Filtering for Digital Signal Processing: MATLAB Applications, Hershey, New York, pp40-45, 2009.

[22] J. Agnew, J. M. Thornton, Just Noticeable and Objectionable Group Delays in Digital Hearing Aids, Journal of the American Academy of Audiology, Vol.11, No. 6, pp330-336, 2000.

[23] K. Tsuneyama, Y. Kiyoki, A Time-Series Phrase Correlation Computing System With Acoustic Signal Processing For Music Media Creation, EMITTER International Journal of Engineering Technology, Vol. 5, No. 1, pp1-15, 2017. 